\title{
Determination of routings and process time information from event logs
}

\author{
Karnok, D.* ,**, Monostori, L.*,** \\ *Computer and Automation Research Institute, Hungarian Academy of Sciences, Budapest, Hungary \\ **Department of Manufacturing Science and Technology, Budapest University of Technology and Economics, Hungary \\ (e-mail:karnok@sztaki.hu)
}

\begin{abstract}
Production planning and scheduling decisions are based on underlying models and their parameters which are typically considered granted, accurate and up-to-date. However, changes and uncertainties may force the production to change, shift and adapt in its settings, consequently, the models and parameters used in the planning and execution phases lose their validity. Usually, these changes are not explicitly known, but are incorporated the manufacturing execution system data. To cope with the missing, uncertain or wrong data, process and data mining should be repeatedly employed on the available manufacturing event logs. The paper describes an analysis framework which is able to extract the required information from large-scale and noisy event logs and offers the ability to infer and restore a more realistic history of the underlying manufacturing process hidden by random noise and consequent errors.
\end{abstract}

\section{INTRODUCTION}

In large-scale, complex and constantly changing manufacturing processes, the process models and parameters used by the planning and execution systems might become out of date relatively frequently. The availability of detailed event $\operatorname{logs}$ from various machines or tracking systems may help automatize the model refinement by using data mining and process mining algorithms. Data and process mining allows the discovery of process routing, correlation between attributes or events, potential hidden states and operations, true task times or machine related attributes. The planning and scheduling steps can be improved by feeding back the values and model changes learnt from the event logs.

However, current process mining methods and tools, usually, cannot handle the simultaneously existing attributes of a real manufacturing process, such as longer loops (e.g., reworks or re-entrant workflow), changes of mid-process product type or process evolution over time. When the manufacturing execution logs are reported manually by the operators on the shop floor, the logged events may contain consequent noise (e.g., misreported machine) atop of the natural variations of the event timing (e.g., completion time variability).

Alternatively, the logged timestamp may represent an 'ownership' time (e.g. setup time or waiting times occasionally included in the time value) instead of a pure task time which makes the real value extraction harder.

Fig.1 displays a typical scenario for differences between a real, non-overlapping batch of tasks and the logged version of the same production process.

The management of any manufacturing process showing the properties above, in terms of algorithms and methods, is nontrivial and prone to biases towards concrete manufacturing areas and companies.

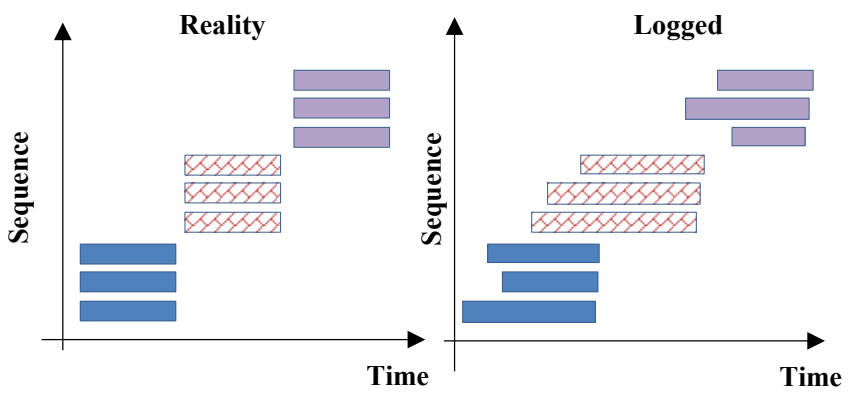

Fig.1. Reality vs. logged events Gantt chart

Based on an earlier work (Karnok, Monostor, 2010), the paper describes extended ideas, methods and algorithms to handle large and complex manufacturing processes which rely heavily on local, shop-floor level decisions. An improved analysis framework, using an iterative, semiautomated approach is proposed to overcome problems in the event $\log$ introduced by the co-existing effects of the manufacturing process, the behavior of the shop-floor personnel or the information systems. Results about the applicability and performance of the newly developed framework are illustrated by using real factory data of a large-scale manufacturer.

\section{RELATED WORK}

The discovery and reverse engineering of manufacturing routing information is closely related to process (or workflow) mining, a variant of data mining where the process structure is learnt from operation event logs (Agrawal, et al., 1998). The idea of using data mining techniques for process mining itself is not new: a method of discovering software processes from event-based data has been proposed earlier, which compared neural network-based, K-tail-based and Markov-based methods in face of noisy process logs (Cook, Wolf, 1996). Petri-nets are a natural way of representing 
process workflows mined from the log data, especially if the underlying process allows parallelism (Weijters, van der Aalst, 2006a). However, certain properties limit the applicability of various process mining algorithms. The log, for example, might contain noise, which can be addressed by using rule-based approach (Maruster, et al., 2006a). There can be other limiting factors that hinder the complete discovery of the workflow, such as the incomplete and nonrepresentative amount of cases in the log (van der Aalst, et. al., 2002a); loops that can emerge from a simpler mining algorithm as parallel operation (Maruster, et al., 2002b). Process mining can also leverage the time information in event logs along with event types (Wen, et al., 2004b) and present interesting performance models (van der Aalst, van Dongen, 2002c). The application of process mining is demonstrated by several real-world examples, including hospitals and other office-like workflows (van der Aalst, et al., 2007a). The field of process mining is still an active research area with various issues and blind spots, such as the re-discoverability problem, quality of the mined workflow model, presence of duplicate tasks, case of non-free-choice (e.g., a branching might depend on pervious states and operations), basic and arbitrary loops, noise types, incompleteness and hidden tasks (van der Aalst, Weijters, 2007a). Free and open source workbenches exist, which allow easy development and testing of new process mining methods, such as ProM (Rozinat, van der Aalst, 2006b).

Data mining techniques are widely used for discovering process related properties, such as cycle time and quality related measures (Gardner, Bieker, 2000). An interesting problem is to mine or discover applied dispatching rules in several-hundred-step long processes of the large-scale manufacturing, especially when the process routing is performed locally on the shop-floor (Li, Olaffson, 2005).

However, large-scale manufacturing often exhibits evolution in terms of process changes. The effect, called concept drift, is known and is a key issue, see (Nishida, 2008a) to name few. Handling concept drift may employ several techniques, such as support vector machines, adaptive ensembles, adaptive decision trees and other change detection-based algorithms (Zliobaite, 2009). In turn, learning and adapting to changes in processes are rarely treated in the literature. Some approaches can be found to detect the process drift when using traditional algorithms, but apart from mining changes from (explicitly stated) change logs (Li, et al., 2008c), no literature trying to address the issue directly is known.

\section{THE ANALYSIS FRAMEWORK}

In order to find the non-stationary routing graph (or net) of various product types from noisy, anomalous operation logs, an iterative framework is proposed, in which partially discovered properties are used for reasoning about the effects present in the logs and confirming or abandoning assumptions about the sources of the anomalies. The analysis framework requires some basic assumptions to be met in the underlying manufacturing process:

- only one operation should be performed on the product at a time in a task (e.g., no parallelism or task overlapping present),
- a machine performs a single operation on a (set of) products at a time.

The framework accepts the logs in two formats:

- as event list, where each log record consists of a timestamp, the type of the event (e.g., start or finish) and the properties of the resources participating in the event (e.g., the product id and type, machine id, operator id, etc.),

- as task list, where each record contains the start and finish timestamps and the properties of the resources associated with the task.

The objective of the framework is to

- build a more detailed model of the manufacturing process in terms of process routing graph, which includes information about loops, reworks, product type changes, scrap-reuse;

- correct various (logging) anomalies in order to restore a more realistic history of the shop-floor manufacturing events; and

- mine the time-related properties of the manufacturing process (e.g., setup times, task times, down times) to allow better key performance indicator evaluations.

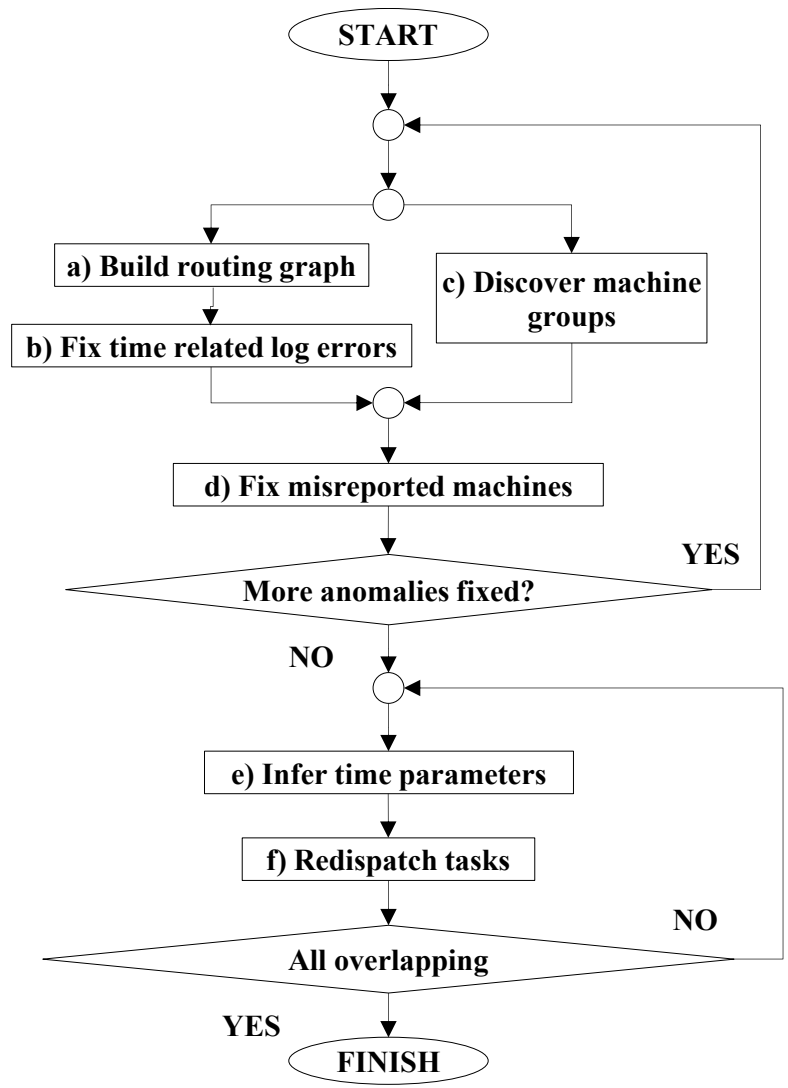

Fig.2. Workflow of the analysis framework

The diagram in Fig 2. shows the log analysis and knowledge discover steps. The steps are described in more detail in the following subsections.

\subsection{Building routing graph (a)}

The first step is to build the routing graphs for various product types and classes (such as regular, calibration, 
experimentation). The global routing graph may be described as a set of nodes and edges. A node contains the class, the product type and operation code along with statistics such as occurrence count in normal and rework cases, occurrence time profile, etc. Edges contain reference to the previous (source) and subsequent (destination) operation nodes and the occurrence count of the particular transition. The transition may indicate product type changes (e.g., source product type unequal to destination product type) and/or class changes (e.g., source class is unequal to destination class).

The log to be analyzed might contain time gaps (log holes): regions in time where no log was gathered, or is simply not available. Detecting gaps like this might be performed by traversing the log and looking for time gaps above a certain threshold (for example 1 day). Some gaps might exist because the entire factory was closed for various reasons (e.g., holiday, full factory maintenance, etc.). The former gap may affect the generated routing graph by introducing random 'jump-ahead' between operations whereas the latter should preserve the continuity without problems (and later on confirmed by using the generated routing itself: no jumpahead). To handle the log holes each individual product's log is split along the boundaries of the holes if necessary and each region is treated as an individual product (with the option to assign a new identifier to the regions if necessary). Artificial start and stop operations are appended to the regions. The artificial operations may help distinguish between product scrapping positions and terminations caused by $\log$ holes or boundaries.

The routing graph is built by traversing each individual product's log records (or a region of it) ordered by the completion timestamps. New nodes are created for product types, class and operation cases unseen before or the statistics of already existing nodes are updated. New edges representing transitions between pairs of nodes are created if necessary or statistics belonging to known transition edges are updated.

The generated routing graph might be overfitting because it may contain nodes or edges derived from noisy or bad log entries. Trimming the graph might be performed using heuristics relying on occurrence (e.g., trim transitions with relative occurrence less than 10\%). However, the start-end continuity-related problems might be fixed directly by using the mined routing graph and heuristics.

\subsection{Fixing time-related log errors (b)}

If several log entries share the same start timestamps and operation but have different finish timestamps, the start timestamp time might be adjusted to the finish timestamp of the previous operation but only if the previous operation in the $\log$ is a 'likely previous' operation (based on the transitions of the routing graph). Otherwise, the entry might be simply deleted.

If a $\log$ record's start timestamp is before the previous record's finish timestamp, a heuristic approach is suggested searching for the previous operation in the log based on the start timestamp. If the appropriate location has been found and that record contains an operation which is a 'likely previous' operation (based on the routing) for the operation in question, the problematic log record might be moved after the location found and the timestamps may be updated to fit into the new destination. If no location is suitable or the timestamp points into a time gap, the record might be deleted as well.

The decision regarding a 'likely previous' test might include the global or relative occurrence of the transition, whether the operation is within a constant number of most frequent previous operation or whether the source or target operation is rework or not.

Non-unique operations and their transition edges mined from the logs might imply loops or shortcuts that could affect the usage of the routing graph in other areas (such as scheduling, simulation or verification based on routing). To avoid cases like this, the operations might be renamed as unique identifiers (create aliases). A simple option is to include the previous original operation identifiers to form a new unique identifier and use that during the route mining process. The number of previous identifiers to use depends on the actual properties of the underlying process and log (e.g., size of loops, the ratio of operation code reuse, etc.).

Another option is to track the so-called trajectory of the incoming and outgoing edges of a node (operation). If the trajectories form an independent (or weakly dependent) group, the node can be split based on the number of groups and the node might be renamed by appending a numerical index to its identifier. The groups of (weakly) independent trajectories could be determined, for example, by using the algorithms for analyzing (strongly) connected components (Hopcroft, Tarjan, 1973).

\subsection{Discovering machine groups (c)}

In order to address the case of different start and end machines reported in the log, the first step is to determine the set of similar machines (machine groups). Some operations might be legally performed on multiple machines of the same kind. One option to discover these machine groups is by using simple text analysis methods on the "names" or "descriptions" of the machines if they are available. Similarly named machines should be capable of performing the same operations. However, if the log lacks textual descriptions or the machines are named in a significantly different way (e.g., machines from different manufacturers), a more thorough analysis is required.

The second option is to aggregate the associations between operations and machines and use some heuristics to define the similarity (e.g. most common operation occurrence on the machines, the relative distribution of the operation among the machines or the relative occurrence of the operation on the potential alternative machines). Consequently, if the textual descriptions of the operations are also available, it is possible to include a text analysis phase involving the descriptions as well. The similarity comparison might be enhanced by making comparisons based on the statistics related to words occurring frequently. Because the accuracy start/end machine might be unreliable, it might be necessary to aggregate the start and end sides separately and only the operations 
occurring on both sides should be considered in the comparisons.

\subsection{Fixing misreported machines (d)}

For the log records which contain different start and end machine, the discovered machine groups might be used for clarifying which attribute should be considered accurate and set the other one accordingly. If the machine group of the input side differs from that of the output side, a simple heuristics might be able to decide which side to use, for example, comparing the relative occurrence of the current operation in the machine groups or using some threshold value for the expected occurrence percentage.

Alternatively, a misreported machine might indicate a missing operation before or after the operation in scope. By using the routing information, the missing operation can be inferred, for example, by checking the previous or next operation occurring the most frequently, and its relation to the misreported machine. If matched, the missing operation can be inserted into the right position.

\subsection{Inferring time parameters (e)}

Extracting time parameters might be trivial in cases when the execution $\log$ truly contains the start and end time of the process by using statistical methods to filter out the small variations in the processing time. However, industrial practice might $\log$ the 'ownership' time instead of just the processing time. Fig.3. shows an example of such kind of time report and its composition.

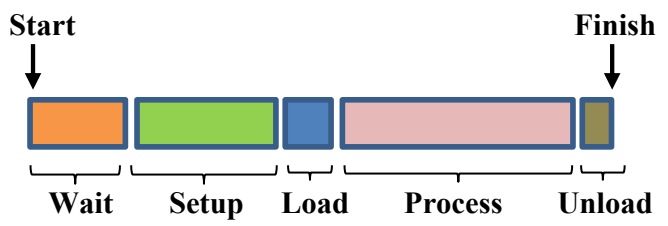

Fig.3. Time composition of the ownership based tracking

Waiting time present when the arriving product on the machine has to wait for some other operation (e.g., previous process, maintenance, setup, etc.) to complete. In the analysis framework, wait time is considered a timespan, where no action is performed in respect to the current batch of products. Setup time is considered when the machine is being prepared to perform the batch in scope. Its numerical value might depend on the machine, the previous operation, the current operation and the product types. Load and unload times are depending on the skillset of the operator and the batch size, but its value might be insignificant in relation to the other time durations of the task. The processing time depends on the product type, the operation, the concrete machine and possibly on the batch size (e.g., an oven is considered independent whereas a conveyor belt is considered dependent). This dependency information if not known a priori can be inferred by searching for an angular pattern in the batch size - logged time diagram for the particular machine and operation.

The potential task overlapping in the reported log might interfere with the reasoning about the time values, therefore, the algorithm primarily looks for cases where there is none or relatively small overlapping present in the logs. These 'clearmoments' are then used as bases when comparing the other instances of the similar cases of the log. In these cases, the process time is considered constant and a weight based heuristic reasoning is performed to separate the setup and process time component. The heuristic considers the start time variation of the task, the location within the current work shift, the amount of the overlapping time with the previous task and the engineering knowledge about the operation at hand. A special case is when no significant difference is present between the scenarios: depending on how the time gap 'behaves', the entire reported time may be considered as the process time or the case is saved for a later iteration when its surrounding environment is potentially cleaned up further. An iteration count is employed to prevent an infinite loop during the evaluation.

The overlapping nature of the log may come from a consistent misreporting of the machine used at the particular operation. Therefore, the algorithm iterates between the time inference and and redispatch (see section 3.6) steps. In each iteration a subset of the cases should become clear and known and are used in cleaning up the rest of the available data.

\subsection{Redispatching tasks (f)}

The objective of the redispatch step is to restore the taskmachine association into a more realistic state. The algorithm assumes that no batches should overlap with the previous or next batch within a particular machine due the physical nature of the manufacturing process. Any overlapping present in the log are considered to be a result of misreporting. After the machine group task associations have been fixed (see section 3.4) the resulting mapping may still be inconsistent with the expectations above. The following scenarios may appear:

- the fix misreported machine step (see section 3.3) corrected the bad machine association in the log record, but it resulted in an overlapping case;

- the task was already overlapping due wrong machine instance was associated from the group;

- the overlapping is constant due consistent misreporting to a single machine and even though more physical alternatives exist, they were basically never present in the log.

An inter-dependency may exist between the correct machine association and task time composition evaluation. On one hand, overlapping might be simply a result of the wait time within a task time composite, or in other hand, the task time evaluat should not even consider a particular task due its false association with the machine being inspected. Therefore, the algorithm in section 3.5 and the algorithm in this section are iterated until all questionable case is resolved or no significant progress can be made. The latter might point to missing environmental data or engineering knowledge about the manufacturing.

The redispatch algorithm works by taking a week worth of tasks associated with the same group of machines and piling them together on a single virtual machine. For each task the 
preferred machine (e.g., the machine named in the log) is kept. The algorithm then iterates over the sequence dimension of batches and based on the overlapping percentage or an already known setup/process time decides whether to keep the task or move it into another virtual machine (see Fig. 4 for example) where it can be settled without overlapping. In case there is no room on any existing virtual machines, a new virtual machine is created.

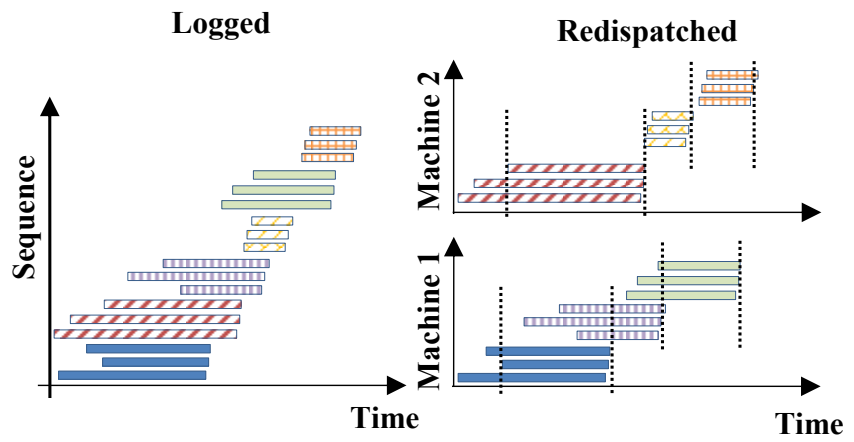

Fig.4. Redispatching the falsely reported tasks

After the task-virtual machine association is established, the algorithm walks the time dimension of all virtual machines and tries to determine the most preferred physical machine instance. Based on this preference, the virtual machine is then re-labeled to that physical machine. The redispatch algorithm tries to move tasks between the now physically labeled machines based on their original preferences but only when there is a big enough time gap on the target physical machine.

\section{CASE STUDY}

The case study is focused on a specific manufacturer's operation logs. Manufacturing is a re-entrant process where a set of operations might be repeated. In addition, product quality is highly sensitive to machine settings, therefore, frequent recalibration is necessary to keep the quality at a certain high level.

Consequently, a constant need for process adjustments exist, which requires experimental runs and might also affect the routing of the regular products in mass production. This creates a product and operation mix and the interleaving of regular, calibration and experimental production runs on many of the machines at the factory. Three kinds of machines are used: single (one product and operation at a time), multi (multiple products in sequence) and batch (multiple products at once). Operators are required to $\log$ the start and completion events for each individual product. The manufacturing technology and the type of the products allow changes of product type during the process in two forms (switching between the versions of the same category or switching to a completely different category), due to some higher level decisions (e.g., unexpected increase of demands), or as results of quality measurements (e.g., high-end vs. lowend market). Engineering knowledge about the actual manufacturing process and operations is rather limited and is present as assumptions. The objective of the study, apart from extracting more detailed routing information, is to clear, correct and reveal these assumptions.
In the logs, each log entry contains the product identifier, current product type; operation code with textual description; the location as sector / cell; a rework indicator and reason; start timestamp, -class, -machine, operator, -process code; end timestamp, -class, -machine, -operator and -process code and a unique sequence number which ensures a global eventorder. The special class attribute indicates whether the performed operation was a regular task, a calibration task or experimentation task. The structure is task-based and can be transformed into an event-log by splitting along the start and end properties and by sharing the common attributes, such as the operation code, location, etc. The analyzed log incorporates about 10 million task entries and 30.000 products out of 100 product types. Products may contain 100 to 600 operations. Unfortunately, the log contains noise and anomalies. The noise affects the start and end timestamps and might cause missing, unnecessary or false data.

Anomalies raise contradiction in the basic assumptions of the manufacturing process and the capabilities of each machine or operator. Ignoring the occurrences of these anomalies would be misleading, as they might be good indications of the real workflow behind the logs and may highlight the (modeling) shortcomings of the software system used for the logging purposes. The following typical anomalies have been identified, and they might be present in the log records at the same time:

- The log records the so-named acquire and release events of the productions. They indicate when the operator has taken or lost ownership of the particular product (instead of when the particular operator has actually started or finished processing on the machine). In addition, depending on the operators' behavior, the reported claim-in time might occur before or after a machine setup has been completed.

- In the concrete manufacturing example multiple parallel operations are not allowed on the same product. A continuity check on the log data revealed that $99 \%$ of the products are affected.

- Cases exist in the log when a product changes its class during its lifetime between regular, calibration or experimental states, sometimes even back and forth. The typical operation sequencing differs considerably in these classes, therefore, a mixture would alter the routing with unnecessary loops or rework cycles.

- Occasionally, the $\log$ records of a product contain a few entries where the logged product type differs from the surrounding entries, jumping back and forth. Consequently, the resulting routing graph might contain unnecessary options for type changes.

- Certain operations are experienced several times in various places within a product's log. However, the operation's textual description suggests that the operation is a common operation, a simple placeholder or a repurposed code introduced to fix the shortcomings of the logging.

- In a longer run, the manufacturing processes related to a product type may change (e.g. new unseen operations occurring in a product's process might be 
discovered in the event logs as time progresses). Operations might become obsolete or operation order might change over time, resulting in unwanted edges in the routing graph.

- The log records may frequently contain different operator and/or machine at the claim-in and claim-out phases of the operation.

The analysis framework from Section 3 was applied to the manufacturing log described in this section. The resulting routing graphs were successfully compared to the reference routing graph provided by the industrial partner and the log anomaly corrections performed and process times extracted were approved and verified by the shop-floor representative from the factory in scope.

The extracted finer routing knowledge may help in several key areas of the manufacturing:

- highlights the misbehavior of the shop-floor personnel in terms of different logging anomalies,

- allows the scheduling system to use a more detailed routing information which includes details of product type changes and reworks,

- allows better what-if simulations to be carried out and provides additional information for factory optimizations,

- makes the automatic maintenance of the manufacturing attributes possible in various shopfloor systems and

- increases the overall transparency of manufacturing and shop-floor activities.

\section{CONCLUSIONS}

In the paper concepts were presented to extract knowledge from large, complex, time-dependent noisy and anomalous process logs in a context of on-going research, aiming at producing accurate and detailed routing graphs, statistics and further anomaly explanations, which, in turn, can help refine models employed by a planning system and reveal modeling or usage issues in the production tracking of a factory.

The analysis framework was applied to a case study with real factory data of a concrete large-scale manufacturer. In general the log files might lack important information (e.g., task start time) or the reasons of certain log anomalies may be completely different in other cases, rendering the corrective measures inert or even corruptive. A different case might require constant and extensive help from an expert of the manufacturer and expect to revalidate and rethink the existing assumptions. The presented framework may be used as a strong base for developing concrete, tailored applications for these new and different cases.

\section{Acknowledgements}

The work was supported by the Hungarian Scientific Research Fund (OTKA) under 'Production Structures as Complex Adaptive Systems’ No. 73376.

\section{REFERENCES}

Karnok D, Monostori L. (2010) Routing model refinement in large scale manufacturing environment by using data mining. In: $43^{\text {rd }}$ CIRP ICMS, 2010:725-734

Agrawal R, Gunopulos D, Leymann F. (1998) Mining process models from workflow logs. Advances in Database Technology EDBT'98. 1998:1377:469-483

Cook JE, Wolf AL (1996) Discovering models of software processes from event-based data. ACM Transactions on Software Engineering and Methodology. 1996:7(3):215249

Weijters T, van der Aalst WMP (2001) Process Mining: Discovering Workflow Models from Event-Based Data. In: 13th Belgium-Netherlands Conference on Artifical Intelligence. 2001:283-290

Maruster, L, Weijters AjMM, van Der Aalst WMP, van der Bosch A (2006a) A rule-based approach for process discovery: dealing with noise and imbalance in process logs. Data Mining and Knowledge Discovery. 2006:13:67-87

van der Aalst WMP, Weijters AJMM, Maruster L (2002a) Workflow mining: which process can be rediscovered? BETA Working Paper Series, WP 74, Eidhoven University of Technology, 2002

Maruster L, Weijters, AJMM, van der Aalst WMP, van der Bosch A (2002b) Process Mining: Discovering direct successors in process logs. In: 5th Int. Conference on Discovery Science, LNAI, 2002:2534:364-373

Wen L, Wang J, van der Aalst WMP, Wang Z, Sun J (2004b) A novel approach for process mining based on event types. BETA Working Paper Series, WP 118, Eindhoven University of Technology, 2004

van der Aalst WMP, van Dongen BF (2002c) Discovering Workflow Performance Models from Timed logs. Int. Conf. on Engineering and Deployment of Cooperative Information Systems (EDCIS). LNCS 2002:2480:45-63

van der Aalst WMP, Reijers HA, Weijters AJMM, van Dongen BF, Alves de Medeiros AK, Song M, Verbeek HMW (2007a) Business Process Mining: An Industrial Application. Information Systems. 2007:32(5):713-732

Rozinat A, van der Aalst WMP (2006b) Decision Mining in ProM. International Conference on Business Process Management. LNCS 2006:4102:420-425

Gardner M, Bieker J. Data Mining Solves Tough Semiconductor Manufacturing Problems (2000) In: the sixth ACM SIGKDD international conference on Knowledge discovery and data mining. 2000:376-383

Li X, Olafsson S. Discovering dispatching rules using data mining (2005) Journal of Scheduling. 2005:8:515-527

Nishida K (2008a) Learning and detecting concept drift. PhD Thesis. Hokkaido University, 2008

Zliobaite I (2009) Learning under concept drift: an overview. Technical Report. Faculty of Mathematics and Informatics, Vilnius University, Lithuania, 2009

Li C, Reichert M, Wombacher A (2008c) Mining based on learning from process change logs. 4th International Workshop on Business Process Intelligence (BPI'08), 2008:121-133

Hopcroft J, Tarjan R (1973) Efficient algorithms for graph manipulation. Communications of the ACM 1973:16:372-378 